\title{
Rapid Acting Insulin Use and Persistence among Elderly Type 2 Diabetes Patients Adding RAI to Oral Antidiabetes Drug Regimens
}

\author{
Usha Sambamoorthi, ${ }^{1}$ Arijita Deb, ${ }^{1}$ Steve Zhou, ${ }^{2}$ Rahul Garg, ${ }^{1}$ Tao Fan, ${ }^{2}$ and Anders Boss ${ }^{2}$ \\ ${ }^{1}$ School of Pharmacy, West Virginia University, Morgantown, WV, USA \\ ${ }^{2}$ Sanofi US, Inc., Bridgewater, NJ, USA \\ Correspondence should be addressed to Usha Sambamoorthi; usambamoorthi@hsc.wvu.edu
}

Received 4 April 2016; Revised 19 August 2016; Accepted 29 August 2016

Academic Editor: Brunella Capaldo

Copyright (C) 2016 Usha Sambamoorthi et al. This is an open access article distributed under the Creative Commons Attribution License, which permits unrestricted use, distribution, and reproduction in any medium, provided the original work is properly cited.

\begin{abstract}
We examined the real-world utilization and persistence of rapid acting insulin (RAI) in elderly patients with type 2 diabetes who added RAI to their drug (OAD) regimen. Insulin-naïve patients aged $\geq 65$ years, with $\geq 1$ OAD prescription during the baseline period, who were continuously enrolled in the US Humana Medicare Advantage insurance plan for 18 months and initiated RAI were included. Among patients with $\geq 2$ RAI prescriptions (RAIp), persistence during the 12-month follow-up was assessed. Multivariate logistic regression analyses identified factors affecting RAI use and persistence. Of 3734 patients adding RAI to their OAD regimen, $2334(62.5 \%)$ had a RAIp during follow-up. Factors associated with RAIp included using $\leq 2$ OADs; cognitive impairment, basal insulin use during follow-up; and higher RAI out-of-pocket costs ( $\$ 36$ to $<\$ 56$ versus $\$ 0$ to $\$ 6.30$ ). Patients were less likely to persist with RAI when on $\leq 2$ OADs versus $\geq 3$ OADs and when having higher RAI out-of-pocket costs ( $\$ 36$ to $<\$ 56$ versus $\$ 0$ to $\$ 6.30$ ) and more likely to persist when they had cognitive impairment and basal insulin use during follow-up. Real-world persistence of RAI in insulin-naïve elderly patients with type 2 diabetes was very poor when RAI was added to an OAD regimen.
\end{abstract}

\section{Introduction}

Rapid acting insulins (RAIs) are characterized by fast-onset (10-15 minutes) and short duration of action (3-5 hours) and are used to manage postprandial glucose excursions in patients with type 2 diabetes [1,2]. Current evidence-based clinical practice guidelines recommend the use of RAI in combination with basal insulin when postprandial glycemic goals are not met with a regimen consisting of basal insulin and oral anti-diabetic drugs (OADs) [3]. However, with the increasing shift towards personalized treatment for the management of type 2 diabetes [3,4], clinicians might resort to approaches that are not suggested in the current guidelines [5]. One such approach is to add RAI to an OAD regimen rather than following the standard practice of adding RAI to basal insulin in order to meet the needs of individual patients. This approach has been shown to be effective in clinical studies [6-8], in which the addition of RAI to an OAD regimen achieved targeted glycemic controls in type 2 diabetes patients. Most of these clinical studies used RAI in combination with metformin $[6,7]$.

Given the need to provide evidence-based personalized treatment, it is important to understand the extent to which RAI is added to OAD regimens and the characteristics of patients who are initiated on RAI and their persistence with this treatment. In addition, as the clinical effectiveness of RAI is tied to persistence, it is critical to understand the factors associated with persistence of RAI when RAI is added to an OAD regimen. A previous study in patients adding RAI to a basal insulin regimen reported poor persistence with RAI [9]. However, the pattern of persistence among type 2 diabetes patients adding RAI to their OAD regimen is unknown.

In the United States, the latest data from the Centers for Disease Control and Prevention suggest that over 25\% of 
the adult population with diabetes is $\geq 65$ years of age [10]. Furthermore, the Medicare-eligible population with diabetes is expected to increase in the coming years $[11,12]$. Therefore, we conducted this study to describe the characteristics of elderly ( $\geq 65$ years of age) Medicare beneficiaries with type 2 diabetes who added RAI to their OAD regimen and to describe the factors associated with RAI continuation and persistence in this patient group.

\section{Methods}

2.1. Study Design. This was an observational, retrospective cohort study using medical, pharmacy, and laboratory claims from US Humana insurance plans for the period 20072012. Humana is an integrated claims database and includes enrollment, medical, and pharmacy claims information for more than 12 million Humana members for both commercial and Medicare advantage plans across the US. For this study, we have only included patients who were enrolled in the Humana Medicare Advantage Prescription Drug plans. The first observed RAI prescription (RAI index date) was used to define the baseline and follow-up periods. The baseline period was 6 months prior to the RAI index date, and the follow-up period was 12 months after the RAI index date.

The study population comprised Medicare beneficiaries with type 2 diabetes who were $\geq 65$ years of age at baseline and had 18-month continuous enrollment (6-month baseline and 12-month follow-up) in Medicare Advantage Prescription Drug (MAPD) plans, had $\geq 1$ OAD claim during the baseline period, and had newly added RAI to their OAD regimen between July 2007 and December 2011. Diagnosis of type 2 diabetes was ascertained using claims for $\geq 1$ inpatient visit or $\geq 2$ physician visits at least 30 days apart and a primary or secondary diagnosis of type 2 diabetes using International Classification of Diseases, 9th Revision, Clinical Modification (ICD-9-CM) codes 250.x0 or 250.x2. Patients were excluded if they used any insulin during the baseline period and were enrolled only in commercial insurance plans or if their gender or insurance plan type was unknown.

2.2. Measures. RAI continuation was measured based on the number of RAI prescriptions during the 12-month followup period. Those with $>1$ RAI prescription during follow-up were considered to have continued with RAI.

Persistence with RAI was examined only in elderly Medicare beneficiaries who had $\geq 2$ RAI prescriptions in the 12-month follow-up period using two measures of persistence based on published methods [9]. Persistence Measure 1 was defined as the absence of any 90-day gap between RAI prescriptions; that is, patients were considered persistent at 12 months if they did not have a 90-day gap by the end of the time period. In persistence Measure 2, persistence at 12 months was defined as having $\geq 4$ RAI prescriptions in the 12 -month follow-up period, with $\geq 1$ RAI prescription in each quarter of the 12-month period. For persistence Measure 2 , the counts of RAI prescriptions included the index RAI prescription.

Independent variables recorded at baseline included the following demographic and clinical characteristics: age, gender, race/ethnicity, region, and insurance plan type (health maintenance organizations [HMOs]; preferred provider organizations (PPOs); and private fee-for-service, [PFFS]/others), any hypoglycemia (identified using ICD-9CM codes 250.8, 251.0, 251.1, and 251.2 for any emergency department [ED], inpatient, or outpatient visits [13]), and severe hypoglycemia (identified using ICD-9-CM codes 250.8, 251.0, 251.1, and 251.2 for inpatient or ED visits [13]), baseline glycosylated hemoglobin (HbAlc) value, diabetes complications using the adapted-Diabetes Complications Severity Index (aDCSI) score [14], healthcare utilization (any inpatient and any ED visit), and medication use (OADs and other concomitant medications). In addition, the following patient complexities specific to the elderly [15] were evaluated: cognitive impairment, depression, injurious falls (ICD-9-CM E-codes [16], and V-codes [17]), urinary incontinence (ICD-9-CM diagnosis codes), and polypharmacy [18]. Polypharmacy was based on the number of different therapeutic drug classes in the 90 days before index period. The definition is based on previous published literature [19]. It was defined as 1 standard deviation (SD) above the mean. Based on our data, those with more than 14 different therapeutic drug classes (mean $=10, \mathrm{SD}=4$ ) during the 90 -day preindex period were considered as having polypharmacy.

The independent variables from the 12-month follow-up period included average RAI out-of-pocket costs (quartiles: $\$ 0$ to $<\$ 6.30 ; \$ 6.30$ to $<\$ 36 ; \$ 36$ to $<\$ 56$; and $\geq \$ 56$ ) and basal insulin use. The RAI out-of-pocket cost was measured as total out-of-pocket cost for RAI prescriptions divided by the total number of RAI prescriptions.

2.3. Statistical Analysis. Chi-square tests were used to assess the unadjusted subgroup differences in RAI continuation and RAI persistence. Multivariable logistic regressions were used for adjusted analyses. Factors used in regression models included number of OADs at baseline, gender, age, race, region, type of insurance plan, any hypoglycemic event at baseline, aDCSI score, HbAlc value at baseline, any inpatient visit, any ED visit, polypharmacy, cognitive impairment, depression, injurious fall, urinary incontinence, basal insulin use at follow-up, and average RAI out-of-pocket costs at follow-up. All analyses were conducted using SAS 9.3 (SAS Institute Inc., Cary, NC, USA).

\section{Results}

3.1. Study Population. A total of 16,850 Medicare beneficiaries $\geq 65$ years of age with type 2 diabetes were identified as receiving RAI therapy between July 2007 and December 2011. Among these patients, 3734 (22.2\%) added RAI to their OAD regimen and were included in this study. The baseline demographic and clinical characteristics of these 3734 patients are presented in Table 1 . The mean age of this population was 72.9 years, $51 \%$ were female, nearly $80 \%$ were white, and $60 \%$ lived in US region South. During the baseline period, $46.2 \%$ were taking $1 \mathrm{OAD}, 40.3 \%$ were taking 2 OADs, and $13.6 \%$ were taking $\geq 3$ OADs. Further, $66 \%$ had an inpatient visit, $60 \%$ had an ED visit, and $14.4 \%$ had polypharmacy. 
TABLE 1: Characteristics of elderly ( $\geq 65$ years) Medicare beneficiaries with type 2 diabetes mellitus who newly added rapid acting insulin to their oral antidiabetic drug regimen. Humana Medicare Advantage Prescription Drug Plan database 2007-2012.

\begin{tabular}{|c|c|c|}
\hline & $N$ & Column\% \\
\hline All & 3,734 & 100 \\
\hline \multicolumn{3}{|l|}{ Number of OADs } \\
\hline $1 \mathrm{OAD}$ & 1,724 & 46.2 \\
\hline 2 OADs & 1,503 & 40.3 \\
\hline$\geq 3$ OADs & 507 & 13.6 \\
\hline \multicolumn{3}{|c|}{ Demographic characteristics } \\
\hline \multicolumn{3}{|l|}{ Gender } \\
\hline Female & 1,899 & 50.9 \\
\hline Male & 1,835 & 49.1 \\
\hline \multicolumn{3}{|l|}{ Race } \\
\hline White & 2,971 & 79.6 \\
\hline African American & 516 & 13.8 \\
\hline Others & 157 & 4.2 \\
\hline Missing & 90 & 2.41 \\
\hline \multicolumn{3}{|l|}{ Age in years } \\
\hline $65-69$ years & 1,296 & 34.7 \\
\hline $70-74$ years & 1,090 & 29.2 \\
\hline $75-79$ years & 764 & 20.5 \\
\hline $80+$ years & 584 & 15.6 \\
\hline \multicolumn{3}{|l|}{ Region } \\
\hline Midwest & 1,122 & 30 \\
\hline South & 2,227 & 59.6 \\
\hline Northeast/west/others & 385 & 10.3 \\
\hline \multicolumn{3}{|c|}{ Health insurance characteristics } \\
\hline \multicolumn{3}{|l|}{ Type of plan } \\
\hline PFFS & 1,311 & 35.1 \\
\hline $\mathrm{HMO}$ & 1,435 & 38.4 \\
\hline $\mathrm{PPO} /$ others & 988 & 26.5 \\
\hline \multicolumn{3}{|c|}{ Baseline clinical characteristics } \\
\hline \multicolumn{3}{|c|}{ Any hypoglycemia } \\
\hline Yes & 469 & 12.6 \\
\hline No & 3,265 & 87.4 \\
\hline \multicolumn{3}{|l|}{ Severe hypoglycemia } \\
\hline Yes & 365 & 9.8 \\
\hline No & 3,369 & 90.2 \\
\hline \multicolumn{3}{|l|}{ aDCSI score } \\
\hline 0 & 926 & 24.8 \\
\hline $1-2$ & 1,141 & 30.6 \\
\hline $3-4$ & 595 & 15.9 \\
\hline $5-13$ & 1,072 & 28.7 \\
\hline \multicolumn{3}{|l|}{$A 1 C$} \\
\hline$<7 \%$ & 377 & 10.1 \\
\hline $7-8 \%$ & 298 & 8 \\
\hline $8-9 \%$ & 234 & 6.3 \\
\hline$>9 \%$ & 332 & 8.9 \\
\hline Not available & 2,493 & 66.8 \\
\hline \multicolumn{3}{|c|}{ Baseline healthcare utilization } \\
\hline \multicolumn{3}{|c|}{ Any inpatient visit } \\
\hline Yes & 2,460 & 65.9 \\
\hline No & 1,274 & 34.1 \\
\hline \multicolumn{3}{|l|}{ Any ER visit } \\
\hline Yes & 2,229 & 59.7 \\
\hline No & 1,505 & 40.3 \\
\hline
\end{tabular}

TABLE 1: Continued.

\begin{tabular}{|c|c|c|}
\hline & $N$ & Column $\%$ \\
\hline \multicolumn{3}{|c|}{ Special conditions for the elderly } \\
\hline \multicolumn{3}{|l|}{ Polypharmacy } \\
\hline Yes $(>14)$ & 537 & 14.4 \\
\hline No $(\leq 14)$ & 3,197 & 85.6 \\
\hline \multicolumn{3}{|c|}{ Cognitive impairment } \\
\hline Yes & 677 & 18.1 \\
\hline No & 3,057 & 81.9 \\
\hline \multicolumn{3}{|l|}{ Depression } \\
\hline Yes & 608 & 16.3 \\
\hline No & 3,126 & 83.7 \\
\hline \multicolumn{3}{|l|}{ Fall } \\
\hline Yes & 236 & 6.3 \\
\hline No & 3,498 & 93.7 \\
\hline \multicolumn{3}{|c|}{ Urinary incontinence } \\
\hline Yes & 173 & 4.6 \\
\hline No & 3,561 & 95.4 \\
\hline \multicolumn{3}{|c|}{ One-year follow-up basal insulin use } \\
\hline \multicolumn{3}{|l|}{ Basal insulin } \\
\hline Yes & 1,973 & 52.8 \\
\hline No & 1,761 & 47.2 \\
\hline \multicolumn{3}{|c|}{ One-year follow-up RAI out-of-pocket cost per RAI prescription } \\
\hline \multicolumn{3}{|c|}{ Average cost per RAI prescription } \\
\hline$\$ 0.0-<\$ 6.3$ & 933 & 25 \\
\hline$\$ 6.3-<\$ 36$ & 945 & 25.3 \\
\hline$\$ 36-<\$ 56$ & 923 & 24.7 \\
\hline$\geq \$ 56$ & 933 & 25 \\
\hline \multicolumn{3}{|c|}{ One-year follow-up RAI out-of-pocket cost per 30-day supply } \\
\hline \multicolumn{3}{|c|}{ RAI OOP cost per 30-day supply } \\
\hline$\$ 0.0-<\$ 6.4$ & 934 & 25 \\
\hline$\$ 6.4-<\$ 35.5$ & 933 & 25 \\
\hline$\$ 35.5-<\$ 47.6$ & 934 & 25 \\
\hline$\geq \$ 47.6$ & 933 & 25 \\
\hline
\end{tabular}

Note: based on 3,734 elderly Medicare beneficiaries aged 65 years and above with type 2 diabetes mellitus, who were continuously enrolled in the Humana Medicare prescription drug plans for 18 months between 2007 and 2011 and added rapid acting insulin to their oral antidiabetic drugs regimen.

A1C: glycated hemoglobin; aDCSI: adapted-Diabetes Complications Severity Index; ER: emergency room; HMO: health maintenance organization; OAD: oral antidiabetic drug; PFFS: private fee-for-service; PPO: preferred provider organization; RAI: rapid acting insulin.

3.2. Basal Insulin Use and RAI Out-of-Pocket Costs over the 12-Month Follow-Up Period. Over the 12-month follow-up period, $52.8 \%$ of the patients who added RAI to their OAD regimen also added basal insulin. Approximately $25 \%$ of patients had RAI OOP cost in each of the four cost quartiles ( $\$ 0$ to $<\$ 6.30 ; \$ 6.30$ to $<\$ 36 ; \$ 36$ to $<\$ 56$; and $\geq \$ 56$ ).

3.3. Continuation of RAI Therapy at the 12-Month Follow-Up. The mean (standard deviation [SD]) number of RAI prescriptions was 3.5 (3.1) at the 12-month follow-up. Of the 3734 patients in the study population, $37.5 \%(n=1400)$ had only 1 RAI prescription (i.e., discontinued RAI therapy), and $62.5 \%$ $(n=2334)$ had $>1$ RAI prescription (i.e., continued RAI 
TABLE 2: Description of elderly ( $\geq 65$ years) Medicare beneficiaries with type 2 diabetes mellitus who newly added rapid acting insulin to their oral antidiabetic drug regimen. Single versus multiple rapid acting insulin prescription during one-year follow-up. Humana Medicare Advantage Prescription Drug Plan database 2007-2012.

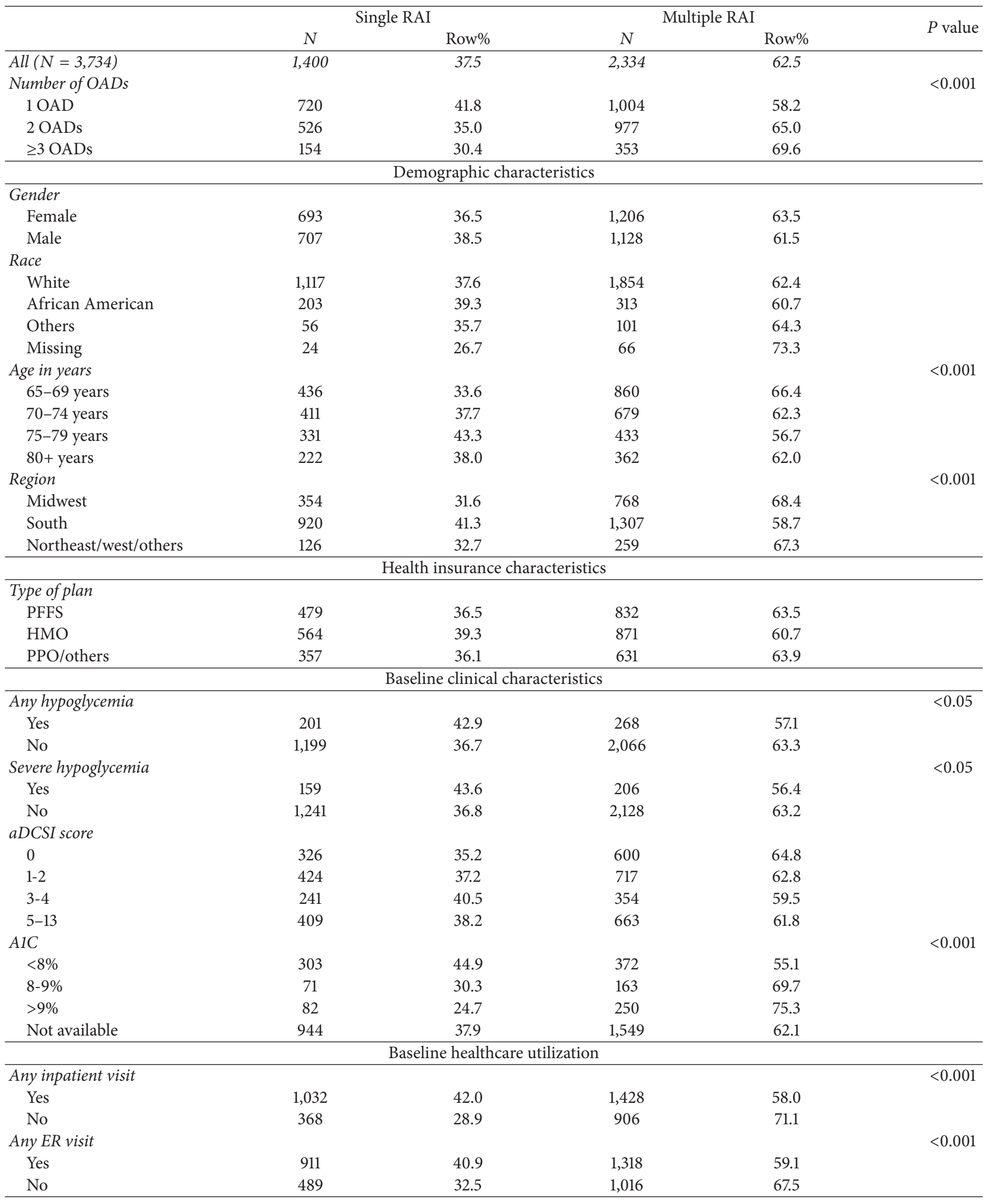


TABLE 2: Continued.

\begin{tabular}{|c|c|c|c|c|c|}
\hline & \multicolumn{2}{|c|}{ Single RAI } & \multicolumn{2}{|c|}{ Multiple RAI } & \multirow{2}{*}{$P$ value } \\
\hline & $N$ & Row\% & $N$ & Row\% & \\
\hline \multicolumn{6}{|c|}{ Special conditions for the elderly } \\
\hline Polypharmacy & & & & & $<0.05$ \\
\hline Yes $(>14)$ & 228 & 42.5 & 309 & 57.5 & \\
\hline No $(\leq 14)$ & 1,172 & 36.7 & 2,025 & 63.3 & \\
\hline Cognitive impairment & & & & & $<0.01$ \\
\hline Yes & 224 & 33.1 & 453 & 66.9 & \\
\hline No & 1,176 & 38.5 & 1,881 & 61.5 & \\
\hline \multicolumn{6}{|l|}{ Depression } \\
\hline Yes & 211 & 34.7 & 397 & 65.3 & \\
\hline No & 1,189 & 38.0 & 1,937 & 62.0 & \\
\hline \multicolumn{6}{|l|}{ Fall } \\
\hline Yes & 96 & 40.7 & 140 & 59.3 & \\
\hline No & 1,304 & 37.3 & 2,194 & 62.7 & \\
\hline \multicolumn{6}{|l|}{ Urinary incontinence } \\
\hline Yes & 63 & 36.4 & 110 & 63.6 & \\
\hline No & 1,337 & 37.5 & 2,224 & 62.5 & \\
\hline \multicolumn{6}{|c|}{ One-year follow-up basal insulin use } \\
\hline Basal insulin & & & & & $<0.001$ \\
\hline Yes & 553 & 28.0 & 1,420 & 72.0 & \\
\hline No & 847 & 48.1 & 914 & 51.9 & \\
\hline \multicolumn{6}{|c|}{ One-year follow-up RAI out-of-pocket cost per RAI prescription } \\
\hline Average cost per RAI prescription & & & & & $<0.001$ \\
\hline$\$ 0.0-<\$ 6.3$ & 308 & 33.0 & 625 & 67.0 & \\
\hline$\$ 6.3-<\$ 36$ & 420 & 44.9 & 516 & 55.1 & \\
\hline$\$ 36-<\$ 56$ & 425 & 45.6 & 507 & 54.4 & \\
\hline$\geq \$ 56$ & 247 & 26.5 & 686 & 73.5 & \\
\hline \multicolumn{6}{|c|}{ One-year follow-up RAI out-of-pocket cost per 30-day supply } \\
\hline RAI OOP cost per 30-day supply & & & & & $<0.001$ \\
\hline$\$ 0.0-<\$ 6.4$ & 283 & 30.3 & 651 & 69.7 & \\
\hline$\$ 6.4-<\$ 35.5$ & 409 & 43.8 & 524 & 56.2 & \\
\hline$\$ 35.5-<\$ 47.6$ & 403 & 43.1 & 531 & 56.9 & \\
\hline$\geq \$ 47.6$ & 305 & 32.7 & 628 & 67.3 & \\
\hline
\end{tabular}

Note: based on 3,734 elderly Medicare beneficiaries aged 65 years and above with type 2 diabetes mellitus, who were continuously enrolled in the Humana Medicare prescription drug plans for 18 months between 2007 and 2011 and added rapid acting insulin to their oral antidiabetic drugs regimen. Significant group differences by multiple versus single prescription for rapid acting insulin were tested using chi-square tests.

A1C: glycated hemoglobin; aDCSI: adapted-Diabetes Complications Severity Index; ER: emergency room; HMO: health maintenance organization; OAD: oral antidiabetic drug; PFFS: private fee-for-service; PPO: preferred provider organization; RAI: rapid acting insulin.

therapy). Subgroup differences were observed between those who had only 1 RAI prescription and those who had $\geq 1$ RAI prescription (continuation) (Table 2).

Multivariable logistic regression analysis showed that continuation of RAI at 12 months (i.e., $\geq 2$ RAI prescriptions at 12 months) was significantly less likely in patients who were taking $1 \mathrm{OAD}$ at baseline compared with those taking $\geq 3$ OADs; were older (aged 70-79 years versus 65-69 years); lived in US region South versus the Northeast/West/other regions; had no diabetes-related complications; had any inpatient visit; and had higher out-of-pocket RAI costs (Table 3).

Continuation with RAI at 12 months was significantly more likely in patients with baseline A1C values $8.0-9.0 \%$ and $>9.0 \%$ compared with $\mathrm{HbAlc}<8.0 \%$, cognitive impairment, and depression and in those who added basal insulin during the 12-month follow-up period (Table 3).

3.4. RAI Persistence. There were significant subgroup differences in the percentage of patients who persisted and did not persist with RAI therapy at 12 months as demonstrated using bivariate analysis (Table 4).

Based on multivariable logistic regression analysis, the baseline factors found to be significantly associated with RAI persistence (Measure 1) at 12 months were cognitive impairment and addition of basal insulin during follow-up. Those patients taking 1 or 2 OADs versus $\geq 3$ OADs at baseline and those having higher OOP costs for RAI prescriptions were significantly less likely to persist (Table 5). Similar findings were observed with RAI persistence Measure 2 (filling $\geq 1$ 
TABLE 3: Adjusted odds ratios and $95 \%$ confidence intervals from logistic regressions of multiple versus single rapid acting insulin prescriptions during 12-month follow-up among elderly ( $\geq 65$ years) Medicare beneficiaries with type 2 diabetes mellitus who added rapid acting insulin to their oral antidiabetic drug. Humana Medicare Advantage Prescription Drug Plan database 2007-2012.

\begin{tabular}{|c|c|c|c|}
\hline & $\mathrm{AOR}$ & $95 \% \mathrm{CI}$ & $P$ value \\
\hline \multicolumn{4}{|l|}{ Number of OADs } \\
\hline $1 \mathrm{OAD}$ & 0.72 & {$[0.58,0.91]$} & $<0.01$ \\
\hline 2 OADs & 0.92 & {$[0.73,1.16]$} & \\
\hline \multicolumn{4}{|l|}{$\geq 3$ OADs } \\
\hline \multicolumn{4}{|c|}{ Demographic characteristics } \\
\hline \multicolumn{4}{|l|}{ Gender } \\
\hline Female & 1.11 & {$[0.96,1.29]$} & \\
\hline \multicolumn{4}{|l|}{ Male } \\
\hline \multicolumn{4}{|l|}{ Race } \\
\hline \multicolumn{4}{|l|}{ White } \\
\hline African American & 0.98 & {$[0.80,1.22]$} & \\
\hline Others & 0.92 & {$[0.64,1.32]$} & \\
\hline Missing & 1.64 & {$[0.99,2.72]$} & \\
\hline \multicolumn{4}{|l|}{ Age in years } \\
\hline \multicolumn{4}{|l|}{ 65-69 years } \\
\hline $70-74$ years & 0.83 & {$[0.70,1.00]$} & $<0.05$ \\
\hline $75-79$ years & 0.71 & {$[0.58,0.87]$} & $<0.001$ \\
\hline $80+$ years & 0.96 & {$[0.76,1.21]$} & \\
\hline \multicolumn{4}{|l|}{ Region } \\
\hline Midwest & 1.05 & {$[0.80,1.36]$} & \\
\hline South & 0.68 & {$[0.53,0.88]$} & $<0.01$ \\
\hline \multicolumn{4}{|c|}{ Northeast/west/others } \\
\hline \multicolumn{4}{|c|}{ Health insurance characteristics } \\
\hline \multicolumn{4}{|l|}{ Type of plan } \\
\hline \multicolumn{4}{|l|}{ PFFS } \\
\hline $\mathrm{HMO}$ & 1.01 & {$[0.84,1.22]$} & \\
\hline PPO/others & 0.98 & {$[0.82,1.18]$} & \\
\hline
\end{tabular}

\begin{tabular}{|c|c|c|c|}
\hline \multicolumn{4}{|c|}{ Baseline clinical characteristics } \\
\hline \multicolumn{4}{|c|}{ Any hypoglycemia } \\
\hline Yes & 0.82 & {$[0.66,1.01]$} & \\
\hline \multicolumn{4}{|l|}{ No } \\
\hline \multicolumn{4}{|l|}{ aDCSI score } \\
\hline 0 & 0.82 & {$[0.66,1.02]$} & $<0.01$ \\
\hline $1-2$ & 0.88 & {$[0.73,1.07]$} & \\
\hline $3-4$ & 0.82 & {$[0.66,1.02]$} & \\
\hline \multicolumn{4}{|l|}{$5-13$} \\
\hline \multicolumn{4}{|l|}{$A 1 C$} \\
\hline \multicolumn{4}{|l|}{$<8 \%$} \\
\hline $8-9 \%$ & 1.48 & {$[1.05,2.07]$} & $<0.05$ \\
\hline$>9 \%$ & 1.94 & {$[1.42,2.65]$} & $<0.001$ \\
\hline Not available & 1.21 & {$[0.99,1.47]$} & \\
\hline \multicolumn{4}{|c|}{ Baseline healthcare utilization } \\
\hline \multicolumn{4}{|c|}{ Any inpatient visit } \\
\hline Yes & 0.54 & {$[0.44,0.65]$} & $<0.001$ \\
\hline \multicolumn{4}{|l|}{ No } \\
\hline \multicolumn{4}{|l|}{ Any ER visit } \\
\hline Yes & 0.90 & {$[0.75,1.07]$} & \\
\hline No & & & \\
\hline
\end{tabular}

TABLE 3: Continued.

\begin{tabular}{|c|c|c|c|}
\hline & AOR & $95 \% \mathrm{CI}$ & $P$ value \\
\hline \multicolumn{4}{|c|}{ Special conditions for the elderly } \\
\hline \multicolumn{4}{|l|}{ Polypharmacy } \\
\hline Yes $(>14)$ & 0.88 & {$[0.72,1.09]$} & \\
\hline \multicolumn{4}{|l|}{ No $(\leq 14)$} \\
\hline \multicolumn{4}{|l|}{ Cognitive impairment } \\
\hline Yes & 1.51 & {$[1.23,1.85]$} & $<0.001$ \\
\hline \multicolumn{4}{|l|}{ No } \\
\hline \multicolumn{4}{|l|}{ Depression } \\
\hline Yes & 1.23 & {$[1.00,1.51]$} & $<0.05$ \\
\hline \multicolumn{4}{|l|}{ No } \\
\hline \multicolumn{4}{|l|}{ Fall } \\
\hline Yes & 0.88 & {$[0.65,1.18]$} & \\
\hline \multicolumn{4}{|l|}{ No } \\
\hline \multicolumn{4}{|l|}{ Urinary incontinence } \\
\hline Yes & 1.08 & {$[0.76,1.52]$} & \\
\hline \multicolumn{4}{|l|}{ No } \\
\hline \multicolumn{4}{|c|}{ One-year follow-up basal insulin use } \\
\hline \multicolumn{4}{|c|}{ Basal insulin } \\
\hline Yes & 2.30 & {$[1.99,2.66]$} & $<0.001$ \\
\hline \multicolumn{4}{|c|}{ No } \\
\hline \multicolumn{4}{|c|}{$\begin{array}{l}\text { One-year follow-up RAI out-of-pocket cost per RAI } \\
\text { prescription }\end{array}$} \\
\hline \multirow{2}{*}{\multicolumn{4}{|c|}{$\begin{array}{l}\text { Average OOP cost per RAI } \\
\text { prescription }\end{array}$}} \\
\hline$\$ 0.0-<\$ 6.3$ & & & \\
\hline$\$ 6.3-<\$ 36$ & 0.55 & {$[0.45,0.67]$} & $<0.001$ \\
\hline$\$ 36-<\$ 56$ & 0.53 & {$[0.43,0.65]$} & $<0.001$ \\
\hline$\geq \$ 56$ & 1.10 & {$[0.89,1.38]$} & \\
\hline
\end{tabular}

Note: based on 3,734 elderly Medicare beneficiaries aged 65 years and above with type 2 diabetes mellitus, who were continuously enrolled in the Humana Medicare prescription drug plans for 18 months between 2007 and 2011 and added rapid acting insulin to their oral antidiabetic drugs regimen. The reference group for the dependent variable is having more than one RAI prescription during the one-year follow-up period.

A1C: glycated hemoglobin; aDCSI: adapted-Diabetes Complications Severity Index; AOR: adjusted odds ratio; CI: confidence interval; ER: emergency room; HMO: health maintenance organization; $\mathrm{OAD}$ : oral antidiabetic drug; PFFS: private fee-for-service; PPO: preferred provider organization; RAI: rapid acting insulin.

RAI prescription every 3 months). Additionally, persistence Measure 2 demonstrated that individuals living in US region South compared with Northeast/West/other regions, and patients with polypharmacy were less likely to persist with RAI (Table 5).

3.5. Sensitivity Analysis. We conducted a sensitivity analysis using an alternative measure of out-of-pocket costs over the 12-month follow-up period. For each person, it was calculated as total out-of-pocket cost for RAI prescriptions divided by the total number of days supplied for RAI prescriptions. This was multiplied by 30 in order to interpret the out-of-pocket cost per 30-day supply. The RAI out-of-pocket cost quartile was as follows: quartiles: $\$ 0$ to $<\$ 6.4 ; \$ 6.4$ to $<\$ 35.5 ; \$ 35.5$ to $<\$ 47.6$; and $\geq \$ 47.6$. 
TABLE 4: Description of elderly ( $\geq 65$ years) Medicare beneficiaries with type 2 diabetes mellitus who newly added rapid acting insulin to their oral antidiabetic drug regimen by rapid acting insulin persistence during one-year follow-up. Humana Medicare Advantage Prescription Drug Plan database 2007-2012 (row\%).

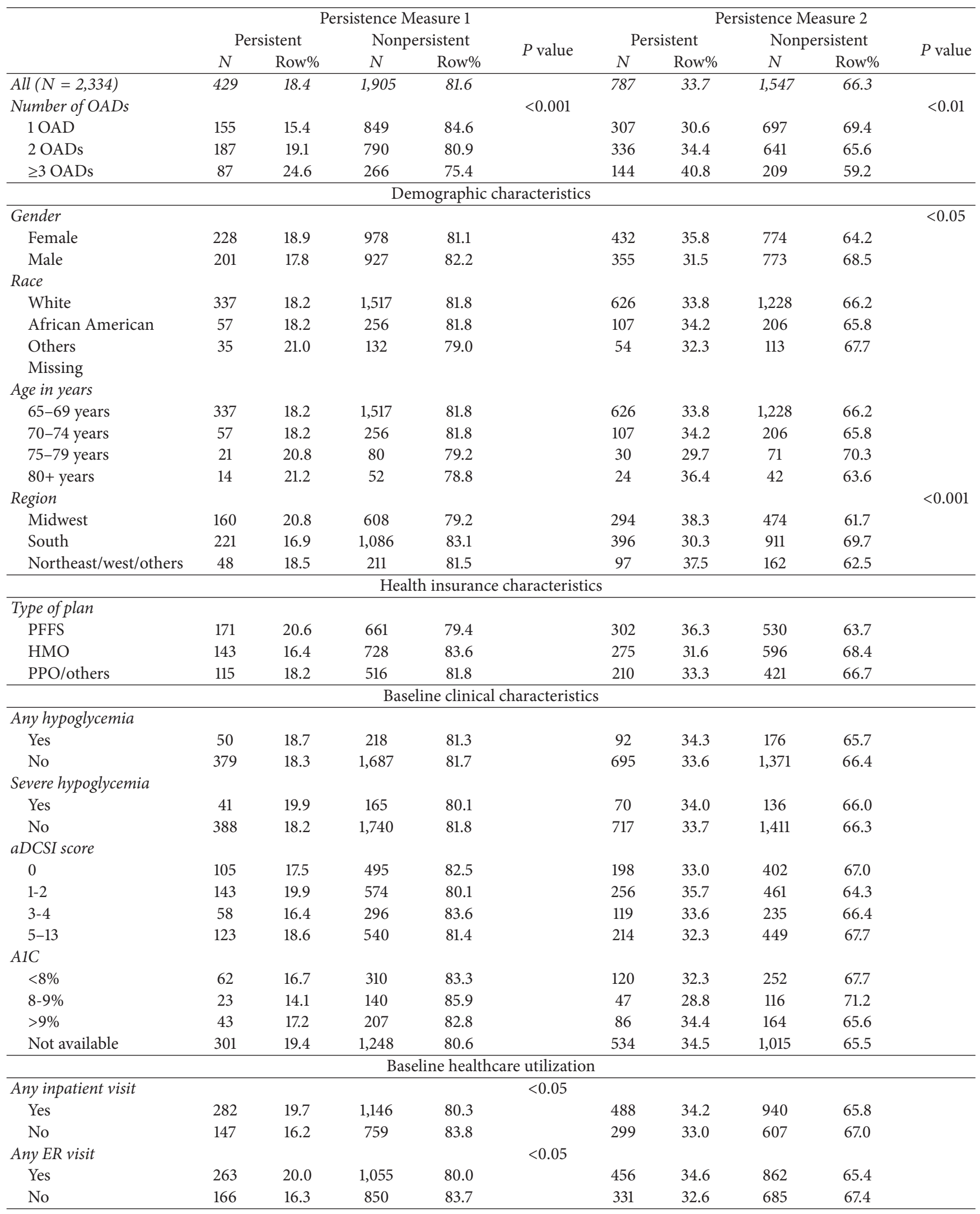


TABLE 4: Continued.

\begin{tabular}{|c|c|c|c|c|c|c|c|c|c|c|}
\hline & \multicolumn{5}{|c|}{ Persistence Measure 1} & \multicolumn{5}{|c|}{ Persistence Measure 2} \\
\hline & \multicolumn{2}{|c|}{ Persistent } & \multicolumn{2}{|c|}{ Nonpersistent } & \multirow{2}{*}{$P$ value } & \multicolumn{2}{|c|}{ Persistent } & \multicolumn{2}{|c|}{ Nonpersistent } & \multirow{2}{*}{$P$ value } \\
\hline & $N$ & Row\% & $N$ & Row\% & & $N$ & Row\% & $N$ & Row\% & \\
\hline \multicolumn{11}{|c|}{ Special conditions for the elderly } \\
\hline \multicolumn{11}{|l|}{ Polypharmacy } \\
\hline Yes $(>14)$ & 50 & 16.2 & 259 & 83.8 & & 90 & 29.1 & 219 & 70.9 & \\
\hline No $(\leq 14)$ & 379 & 18.7 & 1,646 & 81.3 & & 697 & 34.4 & 1,328 & 65.6 & \\
\hline Cognitive impairment & & & & & $<0.001$ & & & & & $<0.001$ \\
\hline Yes & 116 & 25.6 & 337 & 74.4 & & 195 & 43.0 & 258 & 57.0 & \\
\hline No & 313 & 16.6 & 1,568 & 83.4 & & 592 & 31.5 & 1,289 & 68.5 & \\
\hline \multicolumn{11}{|l|}{ Depression } \\
\hline Yes & 86 & 21.7 & 311 & 78.3 & & 139 & 35.0 & 258 & 65.0 & \\
\hline No & 343 & 17.7 & 1,594 & 82.3 & & 648 & 33.5 & 1,289 & 66.5 & \\
\hline Fall & & & & & & & & & & $<0.05$ \\
\hline Yes & 34 & 24.3 & 106 & 75.7 & & 60 & 42.9 & 80 & 57.1 & \\
\hline No & 395 & 18.0 & 1,799 & 82.0 & & 727 & 33.1 & 1,467 & 66.9 & \\
\hline \multicolumn{11}{|l|}{ Urinary incontinence } \\
\hline Yes & 26 & 23.6 & 84 & 76.4 & & 42 & 38.2 & 68 & 61.8 & \\
\hline No & 403 & 18.1 & 1,821 & 81.9 & & 745 & 33.5 & 1,479 & 66.5 & \\
\hline \multicolumn{11}{|c|}{ One-year follow-up basal insulin use } \\
\hline Basal insulin & & & & & $<0.05$ & & & & & $<0.01$ \\
\hline Yes & 280 & 19.7 & 1,140 & 80.3 & & 510 & 35.9 & 910 & 64.1 & \\
\hline No & 149 & 16.3 & 765 & 83.7 & & 277 & 30.3 & 637 & 69.7 & \\
\hline \multicolumn{11}{|c|}{ One-year follow-up RAI out-of-pocket cost per RAI prescription } \\
\hline Average OOP cost & & & & & $<0.001$ & & & & & $<0.001$ \\
\hline$\$ 0.0-<\$ 6.3$ & 112 & 22.0 & 398 & 78.0 & & 180 & 35.3 & 330 & 64.7 & \\
\hline$\$ 6.3-<\$ 36$ & 38 & 16.0 & 200 & 84.0 & & 85 & 35.7 & 153 & 64.3 & \\
\hline$\$ 36-<\$ 56$ & 62 & 8.9 & 636 & 91.1 & & 106 & 15.2 & 592 & 84.8 & \\
\hline$\geq \$ 56$ & 217 & 24.4 & 671 & 75.6 & & 416 & 46.8 & 472 & 53.2 & \\
\hline \multicolumn{11}{|c|}{ One-year follow-up out-of-pocket RAI cost per 30-day supply } \\
\hline OOP cost per 30-day supply & & & & & $<0.001$ & & & & & $<0.001$ \\
\hline$\$ 0.0-<\$ 6.4$ & 171 & 26.3 & 480 & 73.7 & & 281 & 43.2 & 370 & 56.8 & \\
\hline$\$ 6.4-<\$ 35.5$ & 71 & 13.5 & 453 & 86.5 & & 145 & 27.7 & 379 & 72.3 & \\
\hline$\$ 35.5-<\$ 47.6$ & 49 & 9.2 & 482 & 90.8 & & 115 & 21.7 & 416 & 78.3 & \\
\hline$\geq \$ 47.6$ & 138 & 22.0 & 490 & 78.0 & & 246 & 39.2 & 382 & 60.8 & \\
\hline
\end{tabular}

Note: based on 2,334 elderly Medicare beneficiaries aged 65 years and above with type 2 diabetes mellitus, who were continuously enrolled in the Humana Medicare prescription drug plans for 18 months between 2007 and 2011 and added rapid acting insulin to their oral antidiabetic drugs regimen. These individuals had at least 2 claims for rapid acting insulin during the follow-up period. Significant group differences in persistence were tested with chi-square tests. A1C: glycated hemoglobin; aDCSI: adapted-Diabetes Complications Severity Index; ER: emergency room; HMO: health maintenance organization; OAD: oral antidiabetic drug; PFFS: private fee-for-service; PPO: preferred provider organization; RAI: rapid acting insulin.

Results from both bivariate (Tables 2 and 3 ) and multivariate analyses using the alternate definition of RAI outof-pocket suggest that the direction and magnitude of the associations between RAI out-of-pocket costs and RAI continuation and RAI persistence did not change. For example, compared to patients with RAI out-of-pocket cost per 30day supply ranging between $\$ 0$ and $<\$ 6.4$, those with RAI out-of-pocket cost ranging between $\$ 6.4$ and $<\$ 35.5$ ( $\mathrm{AOR}=$ $0.43,95 \% \mathrm{CI}=0.31,0.59$ ) were significantly less likely to be persistent (not shown in Tabular form).

\section{Discussion}

This is the first real-world study using data from an administrative claims database to examine the practice-based addition of RAI to an OAD regimen in elderly patients with type 2 diabetes. Although intensification of OAD therapy with RAI is not a standard of care for postprandial glycemic management in type 2 diabetes, clinical studies have shown the benefits of such practice [6, 7]. Here, we report for the first time practice-based evidence showing that a sizeable proportion of elderly type 2 diabetes patients added RAI therapy to their OAD regimen.

Management of type 2 diabetes patients by treatment intensification of OADs with RAI may be considered in two groups of patients, those in care homes that use RAI as a supplemental dose temporarily and those that use RAI on a regular basis. Clinicians may be uncertain regarding the optimal strategy for insulin intensification [20] or favor resorting to less widely used treatment approaches to meet the needs of individual patients [5], as personalized diabetes management is becoming important. In addition, for patients 
TABLE 5: Adjusted odds ratios and 95\% confidence intervals from logistic regressions of rapid acting insulin persistence during 12-month follow-up among elderly ( $\geq 65$ years) Medicare beneficiaries with type 2 diabetes mellitus who added rapid acting insulin to their oral antidiabetic regimen. Humana Medicare Advantage Prescription Drug Plan database 2007-2012.

\begin{tabular}{|c|c|c|c|c|c|c|}
\hline & \multicolumn{3}{|c|}{ Persistence Measure 1} & \multicolumn{3}{|c|}{ Persistence Measure 2} \\
\hline & AOR & $95 \% \mathrm{CI}$ & $P$ value & AOR & $95 \% \mathrm{CI}$ & $P$ value \\
\hline \multicolumn{7}{|l|}{ Number of OADs } \\
\hline $1 \mathrm{OAD}$ & 0.52 & {$[0.38,0.71]$} & $<0.001$ & 0.63 & {$[0.48,0.81]$} & $<0.001$ \\
\hline 2 OADs & 0.69 & {$[0.51,0.93]$} & $<0.05$ & 0.75 & {$[0.58,0.98]$} & $<0.05$ \\
\hline \multicolumn{7}{|l|}{$\geq 3$ OADs } \\
\hline \multicolumn{7}{|c|}{ Demographic characteristics } \\
\hline \multicolumn{7}{|l|}{ Gender } \\
\hline Female & 0.97 & {$[0.77,1.21]$} & & 1.13 & {$[0.95,1.36]$} & \\
\hline \multicolumn{7}{|l|}{ Male } \\
\hline \multicolumn{7}{|l|}{ Race } \\
\hline \multicolumn{7}{|l|}{ White } \\
\hline African American & 0.93 & {$[0.67,1.30]$} & & 0.98 & {$[0.75,1.29]$} & \\
\hline Others & 1.19 & {$[0.71,1.99]$} & & 0.78 & {$[0.49,1.23]$} & \\
\hline Missing & 0.96 & {$[0.51,1.79]$} & & 0.94 & {$[0.55,1.60]$} & \\
\hline \multicolumn{7}{|l|}{ Age in years } \\
\hline \multicolumn{7}{|l|}{ 65-69 years } \\
\hline $70-74$ years & 0.91 & {$[0.70,1.20]$} & & 1.01 & {$[0.81,1.26]$} & \\
\hline $75-79$ years & 0.94 & {$[0.69,1.29]$} & & 0.96 & {$[0.74,1.25]$} & \\
\hline $80+$ years & 1.02 & {$[0.73,1.44]$} & & 1.20 & {$[0.90,1.59]$} & \\
\hline \multicolumn{7}{|l|}{ Region } \\
\hline Midwest & 1.14 & {$[0.79,1.66]$} & & 1.00 & {$[0.74,1.36]$} & \\
\hline South & 0.91 & {$[0.63,1.31]$} & & 0.70 & {$[0.52,0.94]$} & $<0.05$ \\
\hline \multicolumn{7}{|c|}{ Northeast/west/others } \\
\hline \multicolumn{7}{|c|}{ Health insurance characteristics } \\
\hline \multicolumn{7}{|l|}{ Type of plan } \\
\hline \multicolumn{7}{|l|}{ PFFS } \\
\hline HMO & 0.80 & {$[0.60,1.06]$} & & 0.88 & {$[0.70,1.11]$} & \\
\hline PPO/others & 0.88 & {$[0.67,1.16]$} & & 0.88 & {$[0.70,1.11]$} & \\
\hline \multicolumn{7}{|c|}{ Baseline clinical characteristics } \\
\hline \multicolumn{7}{|l|}{ Any hypoglycemia } \\
\hline Yes & 0.93 & {$[0.65,1.31]$} & & 0.99 & {$[0.75,1.32]$} & \\
\hline \multicolumn{7}{|l|}{ No } \\
\hline \multicolumn{7}{|l|}{ aDCSI score } \\
\hline 0 & 1.10 & {$[0.79,1.55]$} & & 1.08 & {$[0.82,1.43]$} & \\
\hline $1-2$ & 1.20 & {$[0.89,1.60]$} & & 1.19 & {$[0.94,1.52]$} & \\
\hline $3-4$ & 0.80 & {$[0.56,1.14]$} & & 0.98 & {$[0.74,1.30]$} & \\
\hline \multicolumn{7}{|l|}{$5-13$} \\
\hline$A 1 C$ & & & & & & \\
\hline$<8 \%$ & & & & & & \\
\hline $8-9 \%$ & 0.85 & {$[0.50,1.45]$} & & 0.84 & {$[0.55,1.27]$} & \\
\hline$>9 \%$ & 1.02 & {$[0.65,1.60]$} & & 1.07 & {$[0.75,1.53]$} & \\
\hline Not available & 1.08 & {$[0.77,1.49]$} & & 0.98 & {$[0.76,1.28]$} & \\
\hline & & Baseline & e utilizati & & & \\
\hline Any inpatient visit & & & & & & \\
\hline Yes & 1.03 & {$[0.76,1.41]$} & & 0.89 & {$[0.69,1.14]$} & \\
\hline No & & & & & & \\
\hline Any ER visit & & & & & & \\
\hline Yes & 1.12 & {$[0.84,1.49]$} & & 1.00 & {$[0.79,1.26]$} & \\
\hline No & & & & & & \\
\hline
\end{tabular}


TABLE 5: Continued.

\begin{tabular}{|c|c|c|c|c|c|c|}
\hline & \multicolumn{3}{|c|}{ Persistence Measure 1} & \multicolumn{3}{|c|}{ Persistence Measure 2} \\
\hline & AOR & $95 \%$ CI & $P$ value & AOR & $95 \% \mathrm{CI}$ & $P$ value \\
\hline \multicolumn{7}{|c|}{ Special conditions for the elderly } \\
\hline \multicolumn{7}{|l|}{ Polypharmacy } \\
\hline Yes $(>14)$ & 0.71 & {$[0.51,1.01]$} & & 0.73 & {$[0.55,0.96]$} & $<0.05$ \\
\hline \multicolumn{7}{|l|}{ No $(\leq 14)$} \\
\hline \multicolumn{7}{|c|}{ Cognitive impairment } \\
\hline Yes & 1.41 & {$[1.05,1.89]$} & $<0.05$ & 1.50 & {$[1.16,1.92]$} & $<0.01$ \\
\hline \multicolumn{7}{|l|}{ No } \\
\hline \multicolumn{7}{|l|}{ Depression } \\
\hline Yes & 0.99 & {$[0.74,1.34]$} & & 0.84 & {$[0.65,1.08]$} & \\
\hline \multicolumn{7}{|l|}{ No } \\
\hline \multicolumn{7}{|l|}{ Fall } \\
\hline Yes & 1.01 & {$[0.65,1.57]$} & & 1.15 & {$[0.79,1.68]$} & \\
\hline \multicolumn{7}{|l|}{ No } \\
\hline \multicolumn{7}{|c|}{ Urinary incontinence } \\
\hline Yes & 1.12 & {$[0.69,1.81]$} & & 0.98 & {$[0.64,1.48]$} & \\
\hline \multicolumn{7}{|l|}{ No } \\
\hline \multicolumn{7}{|c|}{ One-year follow-up basal insulin use } \\
\hline \multicolumn{7}{|l|}{ Basal insulin } \\
\hline Yes & 1.32 & {$[1.05,1.66]$} & $<0.05$ & 1.33 & {$[1.10,1.60]$} & $<0.01$ \\
\hline \multicolumn{7}{|l|}{ No } \\
\hline \multicolumn{7}{|c|}{ One-year follow-up RAI out-of-pocket cost per RAI prescription } \\
\hline \multicolumn{7}{|c|}{ Average $O O P$ cost per RAI prescription } \\
\hline$\$ 0.0-<\$ 6.3$ & & & & & & \\
\hline$\$ 6.3-<\$ 36$ & 0.48 & {$[0.35,0.66]$} & $<0.001$ & 0.50 & {$[0.38,0.65]$} & $<0.001$ \\
\hline$\$ 36-<\$ 56$ & 0.44 & {$[0.32,0.61]$} & $<0.001$ & 0.42 & {$[0.32,0.56]$} & $<0.001$ \\
\hline$\geq \$ 56$ & 0.41 & {$[0.30,0.55]$} & $<0.001$ & 0.54 & {$[0.42,0.70]$} & $<0.001$ \\
\hline
\end{tabular}

Note: based on 2,334 elderly Medicare beneficiaries aged 65 years and above with type 2 diabetes mellitus, who were continuously enrolled in the Humana Medicare prescription drug plans for 18 months between 2007 and 2011 and added rapid acting insulin to their oral antidiabetic drugs regimen.

A1C: glycated hemoglobin; aDCSI: adapted-Diabetes Complications Severity Index; AOR: adjusted odds ratio; CI: confidence interval; ER: emergency room; HMO: health maintenance organization; OAD: oral antidiabetic drug; PFFS: private fee-for-service; PPO: preferred provider organization; RAI: rapid acting insulin.

with $\mathrm{HbAlc}<8.4 \%$, the postprandial contribution to hyperglycemia is more important than the basal contribution, and therefore, targeting postprandial hyperglycemia with RAI is a relevant option [21]. However, this can result in increased weight gain and hypoglycemia, which is associated with all insulin therapy use but is more pronounced when treatment is intensified with RAI.

A limited number of studies have demonstrated the benefits of adding RAI to an OAD regimen for achieving targeted glycemic control in type 2 diabetes patients [6-8]. The present study did not explore the effect of this approach on $\mathrm{HbAlc}$ levels because baseline and follow-up HbAlc data were not available for all patients.

Having initiated RAI therapy, RAI use was found to be temporary for many of the patients in our study. We report a persistence rate of $18.4 \%$ with persistence Measure 1 in this study. This low persistence rate is similar to that reported in a study that used the same measure to evaluate RAI persistence in an adult type 2 diabetes population when adding RAI to a basal insulin regimen (19.1\%) [9].
There was a significant difference between the two measures of persistence, Measure 1 and Measure 2 (18.4\% versus $33.7 \%$, resp.). The difference between the two measures was not surprising as persistence Measure 1-with a 90-day gap-was more conservative than Measure 2, which had $\geq 1$ prescription per quarter. As physicians often titrate RAI dose, a 90-day gap might not necessarily mean discontinuation of therapy. So a more lenient measure of persistence (Measure 2) is often used.

We identified that baseline use of 1 or 2 OADs versus $\geq 3$ OADs was significantly associated with RAI nonpersistence. Use of fewer OADs at baseline could indicate that the diabetes stage was not very severe in patients, whereas use of $\geq 3$ OADs at baseline may indicate more severe or uncontrolled diabetes that would require treatment intensification with RAI. Patients with high out-of-pocket costs were also significantly less likely to have RAI persistence over a 12 -month period. This is in line with other studies that have also reported high out-of-pocket costs for insulin prescriptions in general [2224] and for RAI specifically, to be a barrier to RAI persistence 
[9]. It has also been suggested that insurance policies that eliminate copays or that lower copays may encourage RAI persistence [22]. Higher RAI out-of-pocket costs with lower RAI continuation and persistence were robust regardless of measures of out-of-pocket costs. These findings have implications for designing value-based insurance to eliminate copays or lower copays in order to encourage RAI persistence.

Interestingly, cognitive impairment is another factor we found to be associated with RAI persistence despite the expectation that this might adversely affect persistence to a drug regimen. It is possible that a patient's cognitive impairment may have been recognized by a family member and/or a healthcare provider, who could have taken steps to ensure that the insulin was administered under supervision, leading to greater persistence among the patients with cognitive impairment. It is plausible that these patients were institutionalized and therefore had caregivers. Further research may elucidate the reasons for greater persistence among the patients with cognitive impairment in our study population.

The augmentation of the RAI plus OAD regimen with basal insulin during the 12-month follow-up was another factor associated with RAI persistence. Basal insulin was initiated by $52.8 \%$ of patients in this study. It is plausible that these patients have needed further intensification of their insulin regimen.

Taken together, these findings suggest that therapies other than RAI plus OADs may be needed for personalized treatment and optimal diabetes care. Further research is required to understand the rationale for adding RAI to OAD regimens in elderly patients with type 2 diabetes, identify the unmet needs of these patients, and assess the comparative effectiveness of different treatment regimens used for personalizing therapy. Future research should also focus on the impact of RAI persistence on clinical outcomes (change in AlC outcomes) and economic outcomes such as diabetes realated healthcare cost and resource utilization.

Limitations. Several limitations of this study should be acknowledged. Coding errors (e.g., under- or overcoding) are a risk when using ICD-9-CM diagnosis codes in medical claims to identify type 2 diabetes, hypoglycemia, and diabetes complications. In addition, because of the methodology used in this study, minor hypoglycemia events may not have been detected. The nature of claims data means that prescriptions rather than actual use of medications were recorded, and RAI prescriptions may not have been added to claims databases. Moreover, the 90-day prescription gap measure of persistence may have underestimated the level of RAI persistence. Some data were unavailable from the database; thus, time from type 2 diabetes diagnosis to RAI initiation could not be controlled as type 2 diabetes diagnosis dates were unavailable, baseline $\mathrm{HbAlc}$ data were not available for the majority of patients, and clinical reasons for RAI initiation could not be examined in this study. Finally, the study included elderly Medicare beneficiaries enrolled in Humana MAPD plans, so the results may not be generalizable to all elderly Medicare beneficiaries with type 2 diabetes.

\section{Conclusions}

A sizeable proportion of elderly patients with type 2 diabetes have RAI added to their OAD regimen. However, use of RAI was temporary, and type 2 diabetes patients' persistence with RAI was very poor. Nonpersistence was associated with use of fewer OADs and higher out-of-pocket costs at baseline, suggesting that patients with less severe diabetes symptoms and those who had to pay extra for their prescription did not persist and that targeting these patient populations through patient education and insurance policies that eliminate copays or lower copays may encourage RAI persistence. Many patients augmented their RAI plus OAD regimen with basal insulin, and this, together with cognitive impairment, was associated with persistence of RAI. Further research to understand the rationale for addition of RAI to OAD among elderly patients with type 2 diabetes is warranted.

\section{Competing Interests}

Steve Zhou, Tao Fan, and Anders Boss are employees of Sanofi US, Inc. Usha Sambamoorthi, Arijita Deb, and Rahul Garg declare that they have no competing interests.

\section{Acknowledgments}

Study funding and editorial support was provided by Sanofi US, Inc. Writing/editorial support for the preparation of this manuscript was provided by Rasilaben Vaghiiani, Ph.D., of Excerpta Medica, funded by Sanofi US, Inc.

\section{References}

[1] L. Monnier and C. Colette, "Addition of rapid-acting insulin to basal insulin therapy in type 2 diabetes: indications and modalities," Diabetes and Metabolism, vol. 32, no. 1, pp. 7-13, 2006.

[2] D. Raccah, "Options for the intensification of insulin therapy when basal insulin is not enough in type 2 diabetes mellitus," Diabetes, Obesity and Metabolism, vol. 10, no. 2, pp. 76-82, 2008.

[3] S. E. Inzucchi, R. M. Bergenstal, J. B. Buse et al., "Management of hyperglycemia in type 2 diabetes, 2015: a patient-centered approach: update to a position statement of the American Diabetes Association and the European Association for the Study of Diabetes," Diabetes Care, vol. 38, no. 1, pp. 140-149, 2015.

[4] S. Subramanian and I. B. Hirsch, "Personalized diabetes management: moving from algorithmic to individualized therapy," Diabetes Spectrum, vol. 27, no. 2, pp. 87-91, 2014.

[5] M. C. Riddle, "The transition from oral agents to combination insulin/oral therapy," in Contemporary Endocrinology: Type 2 Diabetes Mellitus: An Evidence-Based Approach to Practical Management, M. N. Feinglos and M. A. Bethel, Eds., pp. 169181, Humana Press, Totowa, NJ, USA, 2008.

[6] S. Kokić, D. Buković, M. Radman et al., "Lispro insulin and metformin versus other combination in the diabetes mellitus type 2 management after secondary oral antidiabetic drug failure," Collegium Antropologicum, vol. 27, no. 1, pp. 181-187, 2003.

[7] L. Pala, E. Mannucci, I. Dicembrini, and C. M. Rotella, "A comparison of mealtime insulin aspart and human insulin in combination with metformin in type 2 diabetes patients," 
Diabetes Research and Clinical Practice, vol. 78, no. 1, pp. 132135, 2007.

[8] J. Rosenstock, B. Zinman, L. J. Murphy et al., "Inhaled insulin improves glycemic control when substituted for or added to oral combination therapy in type 2 diabetes: a randomized, controlled trial," Annals of Internal Medicine, vol. 143, no. 8, pp. 549-558, 2005.

[9] M. M. Bonafede, A. Kalsekar, M. Pawaskar et al., "Insulin use and persistence in patients with type 2 diabetes adding mealtime insulin to a basal regimen: a retrospective database analysis," BMC Endocrine Disorders, vol. 11, article 3, 2011.

[10] Centers for Disease Control and Prevention, National Diabetes Statistics Report, 2014: Estimates of Diabetes and Its Burden in the United States, Centers for Disease Control and Prevention, Atlanta, Ga, USA, 2014, http://www.cdc.gov/diabetes/ pubs/statsreport14/national-diabetes-report-web.pdf.

[11] K. M. V. Narayan, J. P. Boyle, L. S. Geiss, J. B. Saaddine, and T. J. Thompson, "Impact of recent increase in incidence on future diabetes burden: U.S., 2005-2050," Diabetes Care, vol. 29, no. 9, pp. 2114-2116, 2006.

[12] E. S. Huang, A. Basu, M. O'Grady, and J. C. Capretta, "Projecting the future diabetes population size and related costs for the U.S.," Diabetes Care, vol. 32, no. 12, pp. 2225-2229, 2009.

[13] A. A. Ginde, P. G. Blanc, R. M. Lieberman, and C. A. Camargo Jr., "Validation of ICD-9-CM coding algorithm for improved identification of hypoglycemia visits," BMC Endocrine Disorders, vol. 8, article 4, 2008.

[14] H.-Y. Chang, J. P. Weiner, T. M. Richards, S. N. Bleich, and J. B. Segal, "Validating the adapted diabetes complications severity index in claims data," American Journal of Managed Care, vol. 18, no. 11, pp. 721-726, 2012.

[15] E. Ickowicz, "Guidelines for improving the care of the older person with diabetes mellitus," Journal of the American Geriatrics Society, vol. 51, no. 5, pp. S265-S280, 2003.

[16] S. Mehta, H. Chen, M. L. Johnson, and R. R. Aparasu, "Risk of falls and fractures in older adults using antipsychotic agents: a propensity-matched retrospective cohort study," Drugs \& Aging, vol. 27, no. 10, pp. 815-829, 2010.

[17] M. E. Tinetti, C. Gordon, E. Sogolow, P. Lapin, and E. H. Bradley, "Fall-risk evaluation and management: challenges in adopting geriatric care practices," Gerontologist, vol. 46, no. 6, pp. 717725, 2006.

[18] J. T. Anger, C. S. Saigal, R. Madison, G. Joyce, M. S. Litwin, and Urologic Diseases of America Project, "Increasing costs of urinary incontinence among female medicare beneficiaries," The Journal of Urology, vol. 176, no. 1, pp. 247-251, 2006.

[19] J. F. Goldberg, J. O. Brooks III, K. Kurita et al., "Depressive illness burden associated with complex polypharmacy in patients with bipolar disorder: findings from the STEP-BD," Journal of Clinical Psychiatry, vol. 70, no. 2, pp. 155-162, 2009.

[20] C. Sorli and M. K. Heile, "Identifying and meeting the challenges of insulin therapy in type 2 diabetes," Journal of Multidisciplinary Healthcare, vol. 7, pp. 267-282, 2014.

[21] L. Monnier, H. Lapinski, and C. Colette, "Contributions of fasting and postprandial plasma glucose increments to the overall diurnal hyperglycemia of type 2 diabetic patients: variations with increasing levels of HbAlc," Diabetes Care, vol. 26, no. 3, pp. 881-885, 2003.

[22] M. J. Davies, J. J. Gagliardino, L. J. Gray, K. Khunti, V. Mohan, and R. Hughes, "Real-world factors affecting adherence to insulin therapy in patients with Type 1 or Type 2 diabetes mellitus: a systematic review," Diabetic Medicine, vol. 30, no. 5, pp. 512-524, 2013.

[23] R. Janes, J. Titchener, J. Pere, R. Pere, and J. Senior, "Understanding barriers to glycaemic control from the patient's perspective," Journal of Primary Health Care, vol. 5, no. 2, pp. 114-122, 2013.

[24] S. Nam, C. Chesla, N. A. Stotts, L. Kroon, and S. L. Janson, "Barriers to diabetes management: patient and provider factors," Diabetes Research and Clinical Practice, vol. 93, no. 1, pp. $1-9,2011$. 


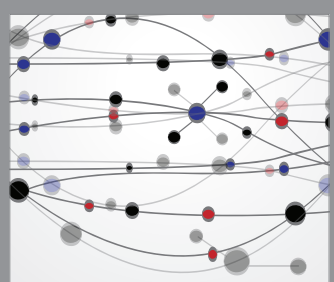

The Scientific World Journal
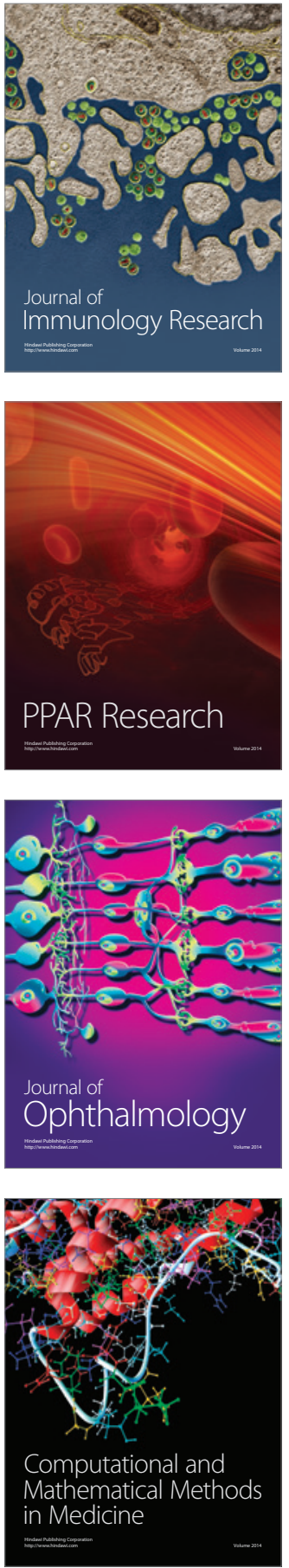

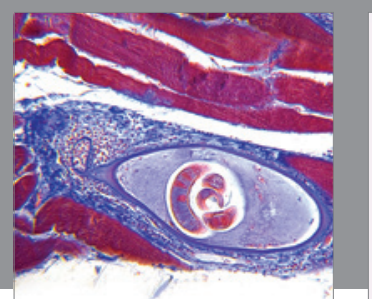

Gastroenterology Research and Practice

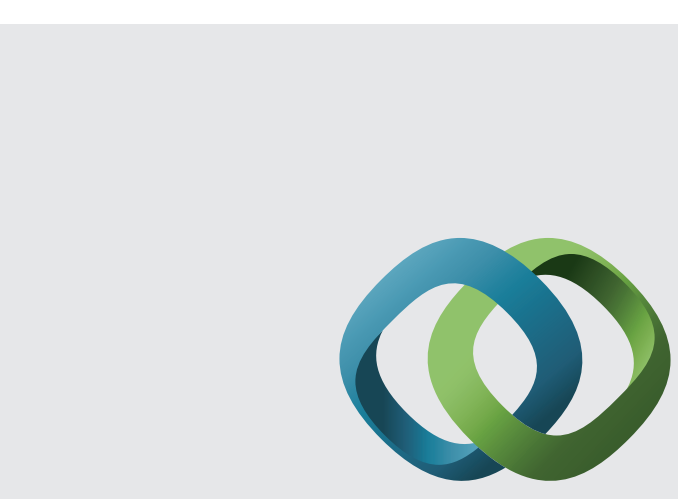

\section{Hindawi}

Submit your manuscripts at

http://www.hindawi.com
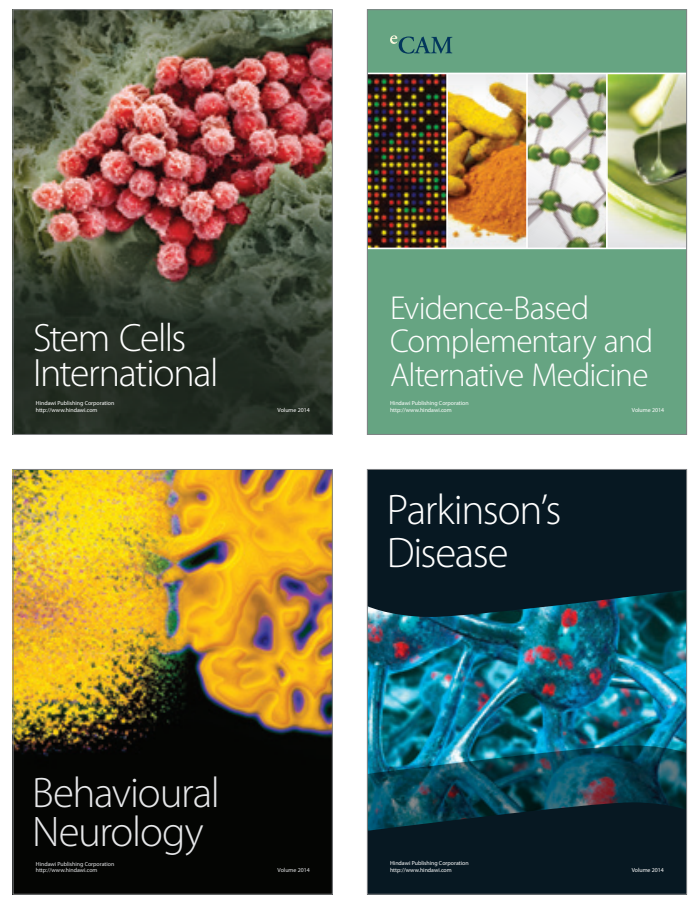
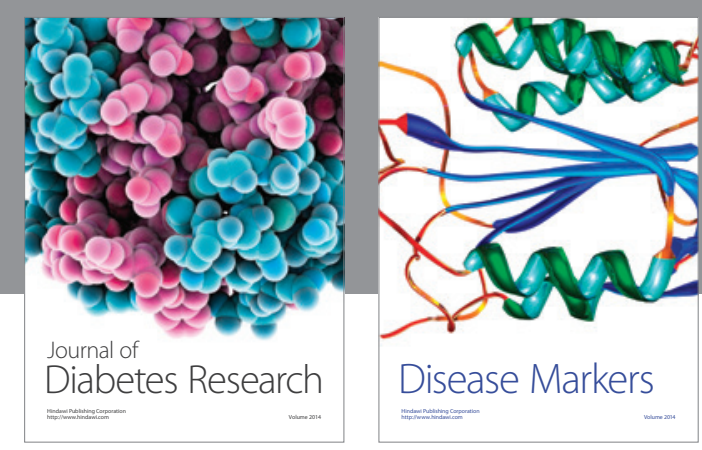

Disease Markers
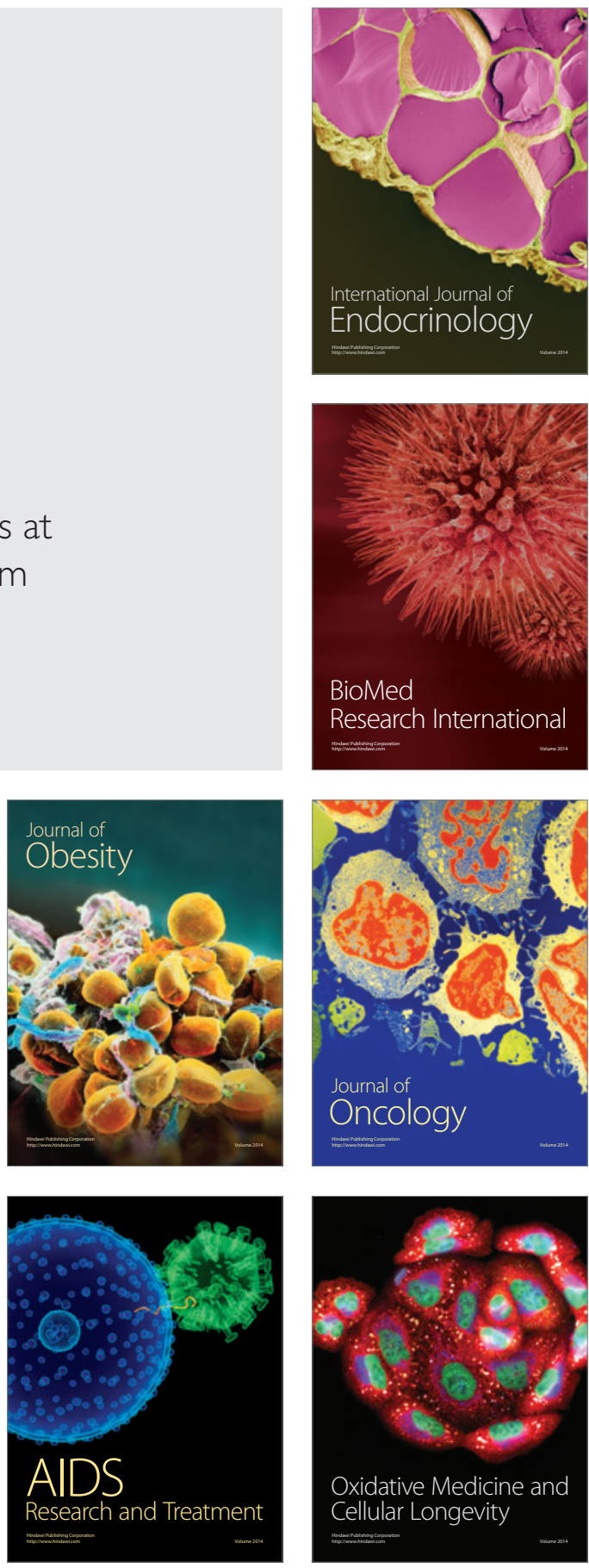